\title{
The Italian Almond Industry: New Perspectives and Ancient Tradition
}

F. Sottile ${ }^{1, \mathrm{a}}$, E. Barone ${ }^{1}$, G. Barbera ${ }^{1}$ and M. Palasciano ${ }^{2}$

${ }_{1}^{1}$ Dipartimento di Scienze Agrarie e Forestali, Università di Palermo, 90128 Palermo, Italy

${ }^{2}$ Dipartimento di Scienze del Suolo della Pianta e degli Alimenti, Università di Bari, 70126 Bari, Italy

Keywords: Italian almond industry, cultivar, rootstocks, planting systems

\begin{abstract}
The almond industry in Italy represents a high degree of structural variability that is determined and sometimes jeopardized by the coexistence of old, traditional orchards and new plantations that differ from each other not only in terms of age but also orchard systems. Apulia and Sicily remain the most important production areas where $90 \%$ of the almond orchards are concentrated. In this southern part of Italy, almond and its derivatives still remain very important. In the last two decades, the Italian almond industry did not show a regular trend, neither with respect to production nor to acreage. An innovative almond industry revealed its consistency year after year since $\mathbf{2 0 0 0}$. Self-fertile cultivars, clonal rootstocks and new planting designs optimized for mechanization were the most important technical aspects which revealed a new way to set up almond orchards. As a matter of fact, the Italian almond industry is still characterized by two strongly different structures: a traditional one based upon old cultivars and orchard systems often with unsatisfactory yields, and an innovative one which is very interesting and appears to be able to partially renew the sector. Technical aspects on this new way to obtain innovative almond orchards and their perspectives are reported here primarily in terms of technical choices.
\end{abstract}

\section{INTRODUCTION}

The Italian almond industry strongly reduced its role at international level in the last decades, from the point of view of both production and acreage. Nevertheless, a strong "traditional almond system" is still widespread even if it is based onto local, nonefficient cultivars and onto old traditional orchard systems that are often impossible to convert to new intensive, specialized systems.

Two southern regions, Sicily (54\%) and Apulia (37\%) together represent more than $91 \%$ of the national almond surface, followed by Sardinia $(3 \%)$ and other southern regions that altogether represent the remaining 6\% (ISTAT, 2010). About 75.000 hectares of almond orchards give a total production of about 110.000 t (Figs. 1 and 2). It's worthwhile to note that the total almond acreage decreased from 296.000 to 75.500 hectares (i.e. a 75\% loss) in the period 1970-2010 (FAOSTAT) while the production was halved (230.600 vs. 108.000 t) (Fig. 2).

This negative trend must be related to the reasons that, as a whole, progressively led to the collapse of the traditional almond industry. Among them, the lack of varietal renovation, the use of obsolete techniques for both plantations and technical management, and poor mechanization are only some of the aspects that should be taken into account to try to understand the reason for this decline and to define possible strategies to regain positions in the international scenario.

The analysis of production trends in the last ten years (Fig. 1) highlights a progressive abandonment of this culture due to alternative uses of land or to alternative cultures. Halfway through the last decade a weak resumption of investments occurred perhaps on the basis of a general reconsideration of almond, as a result of a number of financial support initiatives. Anyway, the decrease in acreage continued till present at an intense rate involving primarily the most traditional and old almond orchards.

afrancesco.sottile@unipa.it 
The Italian almond industry can be described by three different productive configurations. The first one, which is represented by few thousand hectares, has the typical configuration of the modern specialized fully irrigated orchards, with regular spacings and with a consistent suitability for mechanization. The second one is composed by specialized orchards regularly managed but in areas with limited availability of water for irrigation. The last one and still the most widespread, is represented by what can be called traditional almond cultivation, often interspersed with olive trees or other fruit trees, with a very low specialization and a minimal orchard management, particularly in terms of pruning.

The assessment of the state of the Italian almond industry in the two main regions is undoubtedly part of the road map to define possible technical interventions and to suggest any possible growth strategy for the sector.

\section{CURRENT SITUATION}

\section{Cultivar}

The availability of well-suited new fruit trees genotypes of good quality is generally recognized as one of the main factors for the successful establishment of new orchards. For Almond, the traditional cultivars frequently show highly valued pomological traits even if often associated with major limitations such as early bud-break, high percentage of twin fruit, gametophytic incompatibility, high and susceptibility to disease. Furthermore, they are characterized by a very hard stone and, therefore, by a very low kernel yield. Starting from the 1980s, thanks to the new cultivars released by breeding programs carried out in France ('Ferragnès', 'Ferraduel', 'Lauranne', etc.) and in Spain ('Antoñeta', 'Marta', 'Francoli', 'Glorieta', 'Masbovera', etc.), several of the above limitations have been overcome.

In 1993, the Italian Ministry of Agriculture launched a National Project with experimental fields all over the country aimed at the establishment of a comparative evaluation of cultivars and rootstocks for many fruit tree species (Barbera et al., 2001). Five different experimental plots were established in five regions of South Italy (Sicily, Apulia, Basilicata, Calabria and Campania). The vegetative, reproductive and pomological data were periodically collected and evaluated in order to facilitate cultivar and rootstock choice for new almond orchards.

Consistent with the objectives of the almond breeding programs, this National Project has, since the beginning, directed varietal choice toward genotypes well-suited for the exigency of the modern Almond cultivation. Great attention has been devoted to selffertility and late-flowering genotypes, sometimes with some prejudice to fruit quality.

At the top of the list from the five regional working groups are 16 selections including 9 self-fertile and 7 self-incompatible cultivars (Table 1). Among the first group there are 6 Italian cultivars: the Sicilian 'Fellamasa' (intermediate flowering), the Apulian 'Sannicandro' (medium-early flowering), 'Pepparudda' (intermediate flowering), 'Tuono' (medium-late), 'Falsa Barese' and 'Genco' (late flowering), and two new Spanish genotypes ('Francolì' and 'Moncayo', medium-late and late flowering, respectively) and the French genotype ('Lauranne ${ }^{\circledR}$ Avijour', late flowering).

Among the self-incompatible selections, four traditional Italian cultivars are in the list. Big kernels characterize them all and make them especially suited for confetti. Two Sicilian: 'Pizzuta d'Avola', early flowering, and 'Fascionello', medium-early flowering, and two Apulian: 'Fragiulio Grande', intermediate flowering, and 'Trianella', mediumlate. The French 'Ferragnès', late flowering, and two Spanish selections 'Masbovera' and 'Glorieta', medium-late and late flowering, respectively are also included. In addition to the top 16 of the 2013 List, the National Project is now evaluating genotypes recently included in the trials for comparative tests (Palasciano et al., 2013).

Most of new almond acreage in Italy is planted to 'Tuono', a greatly appreciated and highly reliable self-fertile Apulian cultivar. The only defect it presents is the significant percentage of twins but it shows good kernel yield and productivity, especially 
under irrigation. In new plantations 'Tuono' is generally interspersed with 'Genco', which has similar characteristics and with 'Filippo Ceo', also of the traditional Apulian group. However, it should be said that at least in Sicily it is possible to find innovative almond plantations using traditional cultivars such as 'Pizzuta d'Avola', 'Romana' and 'Fascionello', for special utilizations such as confectionery despite their defects.

\section{Rootstocks}

An intensive program of almond rootstock evaluation has never been carried out in Italy. At the end of the 1980s a trial of rootstock evaluation was established in Sicily based upon Monastra's peach $\times$ almond selections (Caboni and Monastra, 1998). However, none of these selections perform better than standard and well known rootstocks.

The Italian Almond industry largely relies on the use of seedling rootstocks. This kind of rootstock is still the most utilized in new plantations. For this purpose, the cultivar 'Don Carlo' offers uniform, fast growing seedlings. More recently, some plantations have been established primarily in Sicily using the GF677 peach $\times$ almond hybrid in specialized orchards under irrigation (Godini et al., 1998). As a whole, they show a slight lack of uniformity between scion and rootstock in the first years, though this is no longer true after 4-5 years.

Some other rootstocks have been proposed as alternatives to GF677 for specific purposes. Among them Penta and Tetra (P. domestica L.) seem to be more suitable for plantations in areas subjected to flooding (Nicotra and Moser, 1997). However, little is known with reference to trunk stability due to the relatively shallow root system, especially where machine-harvested.

\section{Planting Systems}

Italian traditional almond cultivation has been interspersed with other fruit trees so that uneven spacing and irregular orchard design are normal. There is also a kind of specialized almond plantation in which wide spacings and free training systems with irregular pruning are adopted under rainfed conditions.

In contrast, new orchards are also planted using a number of modern and rational orchard management practices. Densities are between 300 and 400 trees per hectare $(6 \times 6$ $-5 \times 5 \mathrm{~m}$ ), depending to soil fertility and to the different type and degree of mechanization adopted.

The only training system adopted is the free-vase with $4-5$ branches at $80-90 \mathrm{~cm}$ from the ground. Pruning is performed yearly in order to keep the tree inside the allotted space, to avoid excess vegetation in the top of the canopy, and to make possible an adequate shoot formation at the base of the canopy and a suitable shape for mechanical harvest.

Drip ( 2 points/tree) is the main irrigation system. Both in Sicily and in Apulia, the adopted volumes are generally below $1.000 \mathrm{~m}^{3}$ per hectare. The amount of water to apply is mainly determined by the stage of fruit growth in order to optimize seed fill. Trials carried out in Sicily have shown that with this kind of irrigation, it is possible to obtain a significant improvement of 'Tuono' kernel yield (45 vs. $34-36 \%$ in rainfed conditions) (Barbera et al., 1993).

\section{Fruit Ripening and Postharvest Management}

In the new plantations, trunk shakers are used, while in the traditional plantations trees are harvested manually with the help of reeds or perches. Trunk shakers are preferred to limb shakers. Trunk shakers can also be utilized for other tree species such as olive or pistachio but, due to their cost, only few growers own them. Other equipment is required for the postharvest handling of the fruit.

Only very few growers carry out mechanical pruning. With the exception of some specific conditions, it remains poorly adopted.

Hulling and drying is carried out 24-48 h after harvest. Some growers store nuts 
in-shell. Almonds are marketed mainly as whole kernels but can also be peeled or further processed to obtain special products for the confectionery and agro-food industry.

\section{CONCLUSIONS}

Due to the complexity of the almond industry in Italy, it is hard to indicate the perspective of the sector as a whole. As with other traditional Mediterranean fruit tree species such as olive, it is difficult to imagine the future survival of a large proportion of these plantations. However, they provide other services to the community and regions (protection against soil erosion, landscape, carbon dioxide sequestration and so on). Furthermore, these old plantations represent a valuable source of genetic diversity that is worthwhile to preserve. As a matter of fact, this kind of almond cultivation will survive only if helped by specific programs of maintenance. On the other hand and from the market point of view, it is not possible to predict the significance of the role of the Italian Almond industry in the international scenario even when this long phase of modernization of the sector is complete.

\section{Literature Cited}

Barbera, G., Catania, F., Godini, A., Insero, O., Mattatelli, B., Palasciano, M. and Senesi, E. 2001. The Italian almond evaluation project. Options Méditerranéennes N. 56:123128.

Barbera, G., La Mantia, T., De Palma, L., Monastra, F. and Schirra, M. 1993. Response of Ferragnes and Tuono almond cultivars to different environmental conditions in Southern Italy. Acta Hort. 373:125-128.

Caboni, E. and Monastra, F. 1998. New selections of almond, putative rootstocks for peach and almond. Cahiers Options Méditerranéennes N. 33:157-162.

Godini, A. and Palasciano, M. 1998. Growth and yield of four self-fruitful and four selfunfruitful almonds on three rootstocks: a thirteen-year study. Acta Hort. 470:200-207.

Nicotra, A. and Moser, L. 1997. Two new plum rootstocks for peach and nectarines: penta and tetra. Acta Hort. 451:269-272.

Palasciano, M., Sottile, F., Lipari, E. and Del Signore, M.B. 2013. Le varietà per i nuovi impianti di mandorlo 2013. L'Informatore Agrario 22:36-38. 


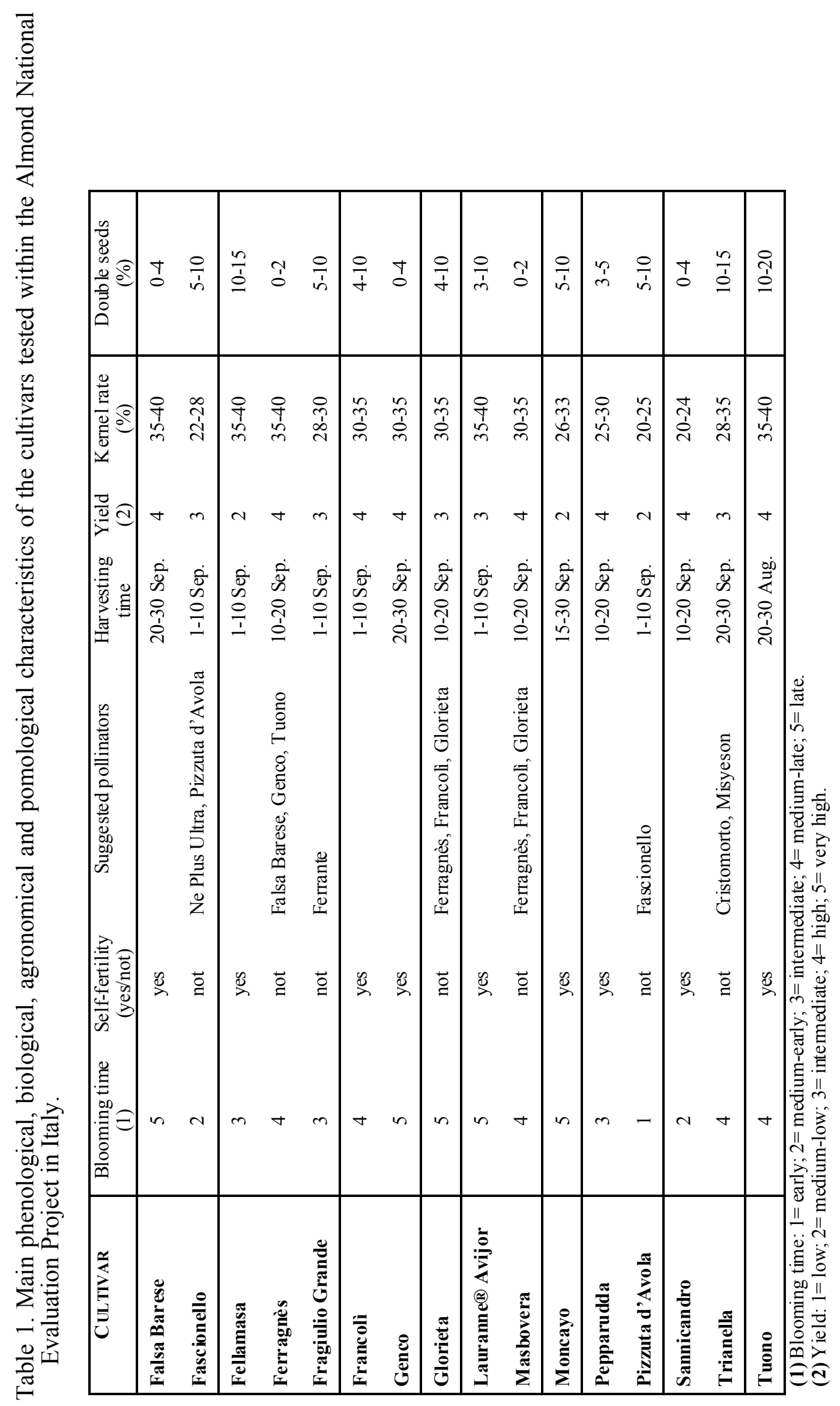




\section{Figures}

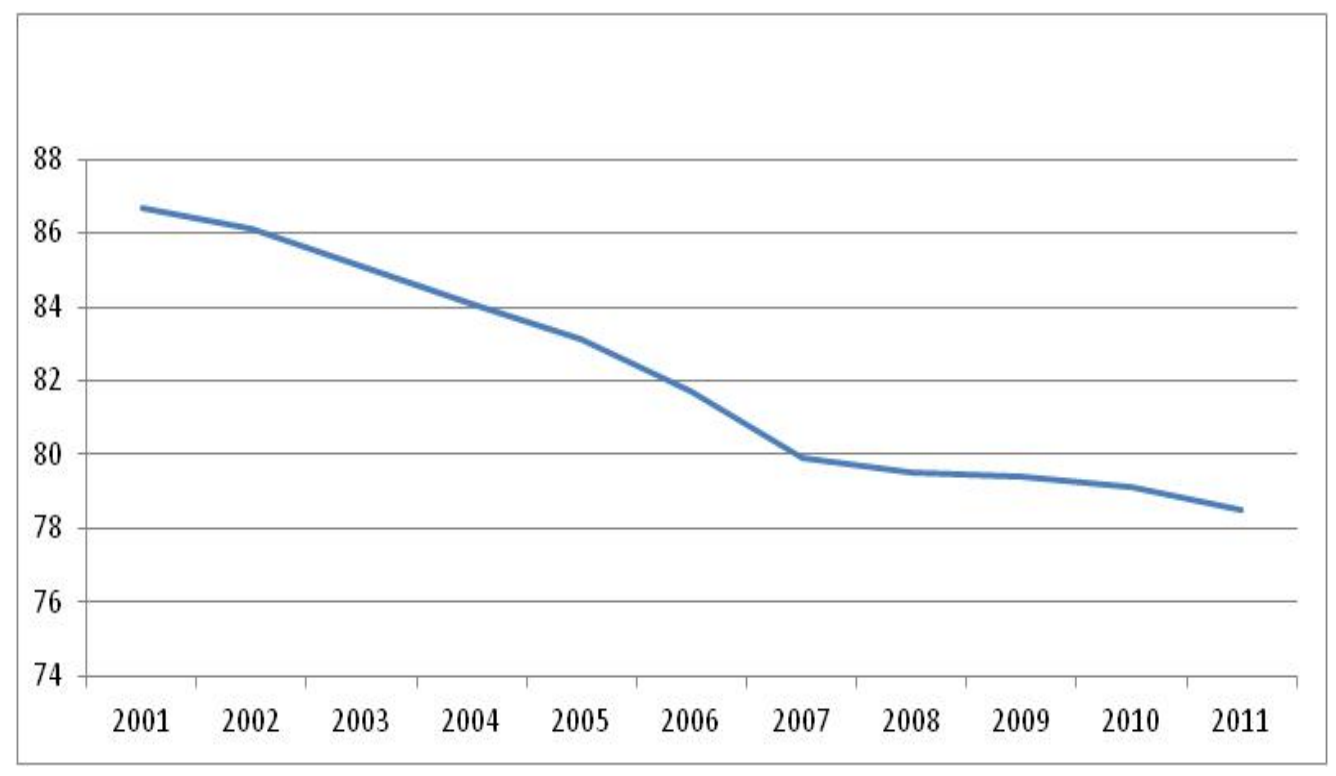

Fig. 1. Area harvested (thousand ha) of almond orchards in Italy (faostat.fao.org).

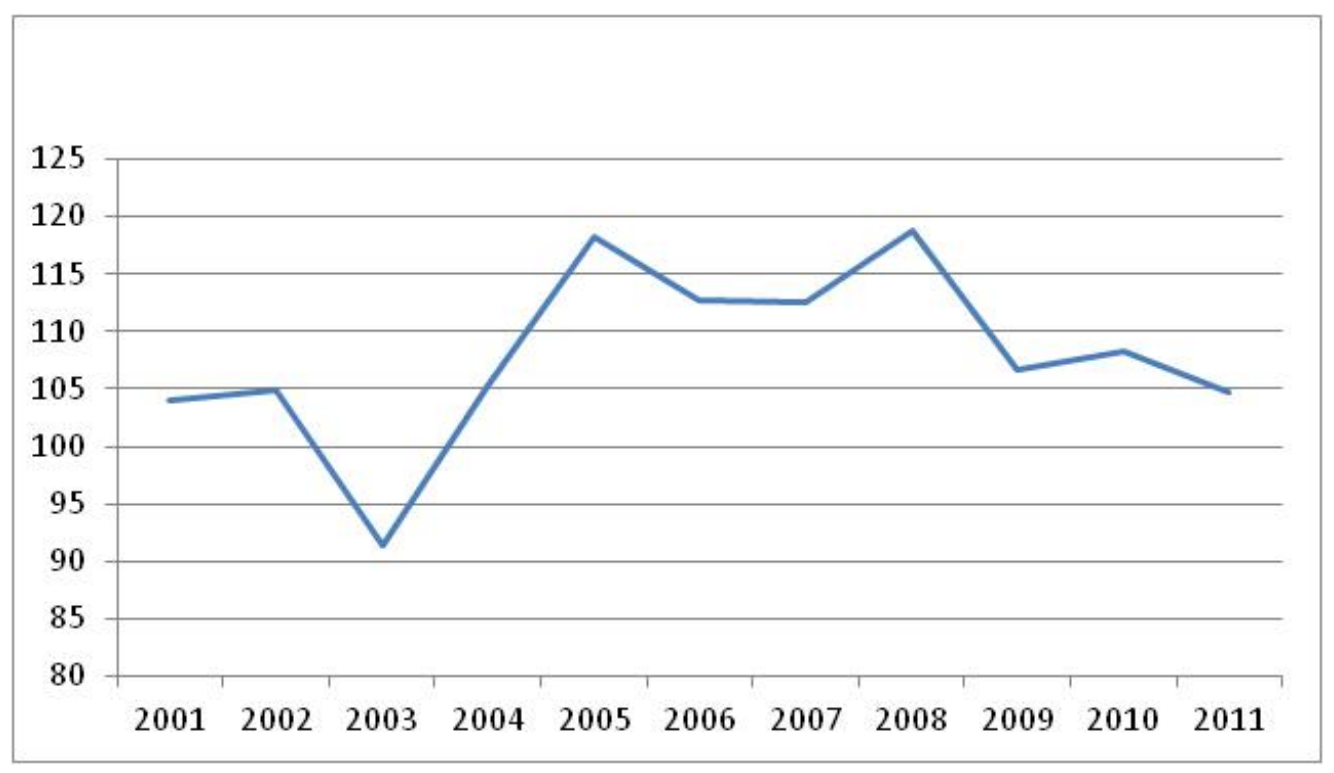

Fig. 2. Almond production (thousand tons) in Italy (faostat.fao.org). 